\title{
Avoiding cosmological oscillating behavior for $S$-brane solutions with diagonal metrics
}

\author{
V. D. Ivashchuk* and V. N. Melnikov ${ }^{\dagger}$ \\ Center for Gravitation and Fundamental Metrology, VNIIMS, Institute of Gravitation \\ and Cosmology, Peoples' Friendship University of Russia, 46 Ozernaya Street, G-361, Moscow, 119361, Russia \\ D. Singleton \\ Department of Physics, California State University Fresno, Fresno, California 93740-8031 USA \\ and Escuela de Fisica, Universidad de Costa Rica San Jose, Costa Rica
}

(Received 1 September 2005; published 11 November 2005)

\begin{abstract}
In certain string inspired higher dimensional cosmological models it has been conjectured that there is generic, chaotic oscillating behavior near the initial singularity - the Kasner parameters which characterize the asymptotic form of the metric jump between different, locally constant values and exhibit a neverending oscillation as one approaches the singularity. In this paper we investigate a class of cosmological solutions with form fields and diagonal metrics which have a maximal number of composite electric $S$ branes. We look at two explicit examples in $D=4$ and $D=5$ dimensions that do not have chaotic oscillating behavior near the singularity. When the composite branes are replaced by noncomposite branes chaotic oscillating behavior again occurs.
\end{abstract}

DOI: 10.1103/PhysRevD.72.103511

PACS numbers: 98.80.Cq, 04.50.+h, 05.45.-a

\section{INTRODUCTION}

In [1-6] the behavior of certain multidimensional solutions with block-diagonal metrics was examined in terms of the billiard representation in generalized Lobachevsky spaces. The billiard representation is a graphical method of studying the asymptotical motion of the point described by the anisotropic minisuperspace coordinates, $z^{a}(t)$, in a region enclosed by "hard walls" which come from the matter sources. The point with coordinates, $z^{a}(t)$, moves inside this region until it encounters a "wall" at which point it recoils according to the reflection law moving toward another wall in the manner of a billiard ball on a billiard table. It was shown that certain multidimensional cosmological solutions exhibit oscillating behavior near the singularity in the sense that the anisotropic part of minisuperspace coordinate functions, that characterize the solutions, exhibit a never-ending oscillation as one approaches the singularity. The first example of such behavior near the singularity was discovered in a 4dimensional Bianchi-IX cosmological, "mixmaster" model [7] by Belinskii, Lifshitz, and Khalatnikov in [8].

The "billiard" description of this behavior in the 4D case was suggested by Chitre in [9]. More recent publications can be found in [10-13]. In [1-3,14] this billiard approach of Chitre was generalized to the multidimensional case.

In the case of multidimensional gravity with either "perfect-fluid" or scalar and form fields, one can give conditions under which one does or does not get never-ending oscillating behavior near the singularity. Here we give a

\footnotetext{
*Electronic address: rusgs@phys.msu.ru

†Electronic address: melnikov@phys.msu.ru

‡Electronic address: dougs@csufresno.edu
}

brief summary of these conditions. In [3] a detailed description of the billiard approach for the multidimensional model with an $m$-component perfect-fluid matter source was given. This model was defined on the manifold

$$
M=\mathbf{R} \times M_{1} \times \ldots \times M_{n},
$$

with the block-diagonal metric described by the line element

$d s^{2}=-\exp [2 \gamma(t)] d t^{2}+\sum_{i=1}^{n} \exp \left[2 x^{i}(t)\right] g_{m_{i}, n_{i}}^{(i)}\left(y_{i}\right) d y_{i}^{m_{i}} d y_{i}^{n_{i}}$

where the manifold $M_{i}$ with the metric $g^{(i)}$ is an Einstein space of dimension $d_{i}, i=1, \ldots, n ; n \geq 2$. The matter sources had an equation of state with the pressures being proportional to the densities

$$
p_{i}^{(\nu)}=\left(1-\frac{u_{i}^{(\nu)}}{d_{i}}\right) \rho^{(\nu)}
$$

where $u_{i}^{(\nu)}=$ const, with $i=1, \ldots, n$ and $\nu=1, \ldots, m$. It was also assumed that

$$
\sum_{i=1}^{n} u_{i}^{(\nu)}>0
$$

for all $\nu$. The densities were restricted to be positive definite, $\rho^{(\nu)}>0$, for any component with a positive square of the vector $u^{(\nu)}$ defined with respect to the dual minisupermetric as

$$
\left(u^{(\nu)}\right)^{2}=\sum_{i=1}^{n} \frac{\left(u_{i}^{(\nu)}\right)^{2}}{d_{i}}+\frac{1}{2-D}\left(\sum_{i=1}^{n} u_{i}^{(\nu)}\right)^{2}>0,
$$

where $D$ is total dimension of $M$ in (1). 
Using the billiard approach [1,2] it was shown in [3] that the solutions to higher dimensional Hilbert-Einstein equations with pressures and densities satisfying the above conditions had asymptotical Kasner-like behavior as $\tau \rightarrow$ +0 ,

$$
d s_{a s}^{2}=-d \tau^{2}+\sum_{i=1}^{n} A_{i} \tau^{2 \alpha^{i}} g_{m_{i}, n_{i}}^{(i)}\left(y_{i}\right) d y_{i}^{m_{i}} d y_{i}^{n_{i}},
$$

when there existed Kasner parameters in (6) obeying the following two relations:

$$
\begin{gathered}
\sum_{i=1}^{n} d_{i} \alpha^{i}=1, \\
\sum_{i=1}^{n} d_{i}\left(\alpha^{i}\right)^{2}=1
\end{gathered}
$$

and the following inequalities were valid,

$$
\sum_{i=1}^{n} u_{i}^{(\nu)} \alpha^{i}>0, \quad \text { if }\left(u^{(\nu)}\right)^{2}>0 .
$$

Here $\tau$ is the proper time given by $d \tau^{2}=e^{2 \gamma(t)} d t^{2}$ and all $A_{i}>0$. When the condition (9) was not satisfied, namely, if for any Kasner set of parameters $\alpha$ there existed a fluid component with index $\nu_{0}$ [obeying $\left(u^{\left(\nu_{0}\right)}\right)^{2}>0$ ], such that

$$
\sum_{i=1}^{n} u_{i}^{\left(\nu_{0}\right)} \alpha^{i} \leq 0
$$

one finds never-ending oscillating behavior near the singularity similar to that of the Bianchi-IX model.

In [5] the above analysis was repeated but with the matter sources given by scalar fields and form fields. The action for this system was given by

$$
\begin{aligned}
S_{g}= & \int d^{D} x \sqrt{|g|}\left(R[g]-h_{\alpha \beta} g^{M N} \partial_{M} \varphi^{\alpha} \partial_{N} \varphi^{\beta}\right. \\
& \left.-\sum_{a \in \Delta} \frac{\theta_{a}}{n_{a} !} \exp \left[2 \lambda_{a}(\varphi)\right]\left(F^{a}\right)^{2}\right)
\end{aligned}
$$

where $g$ is the multidimensional metric with the line element $g_{M N}(x) d x^{M} d x^{N}, \varphi=\left(\varphi^{\alpha}\right) \in \mathbf{R}^{l}$, is a vector of scalar fields, and $F^{a}=d A^{a}$ is an $n_{a}$-form field. In (11) $h_{\alpha \beta}$ is a constant, symmetric, positive definite, $l \times l$ matrix, and $n_{a} \geq 1, \theta_{a}= \pm 1$. Also in (11) $\lambda_{a}(\varphi)=\lambda_{a \alpha} \varphi^{\alpha}$ gives the coupling between the dilaton and form fields, $a \in \Delta$ with $\Delta$ being some finite set, and $\alpha=1, \ldots, l$. The set $\Delta$ can be thought of as a set of "color" indices.

In [5] the form fields in (11) were taken to have an electric, composite brane ansatz, e.g. describing $S$ branes (or spacelike branes). Previous, related work on cosmological and $S$-brane solutions can be found in [15-33] and references therein. For a $(p+2)$-form field this composite ansatz is written as $F=\sum_{I \in \Omega_{e}} d \Phi^{I} \wedge \tau(I)$ where $\Phi^{I}$ is a scalar potential, $\Omega_{e}$ is the set of multi-indices of the form $\left\{i_{0}, i_{1}, \ldots, i_{p}\right\}$ with $i_{k}$ being a number in the set $(1, \ldots, n)$, and $\tau(I)$ is a $p+1$ volume form. A noncomposite ansatz would involve only a single term with no sum, i.e. $F=$ $d \Phi^{I} \wedge \tau(I)$. More details on the electric, composite ansatz are given in the next section. Using this electric, composite ansatz some special classes of cosmological solutions where found on the manifold (1) and a billiard representation near the singularity was obtained, when certain restrictions on the brane configuration were imposed - these included restrictions on brane intersections that guaranteed the block diagonality of the stress-energy tensor (see also [17]). Using this representation it was shown that under certain conditions the solutions under consideration had a metric in (2) with asymptotic Kasner behavior as in (6) and with the scalar fields of the asymptotic form

$$
\varphi_{a s}^{\beta}=\alpha^{\beta} \ln \tau+\text { const, }
$$

as $\tau \rightarrow+0$. The set of Kasner parameters for the metric and scalar fields, $\alpha=\left(\alpha^{A}\right)=\left(\alpha^{i}, \alpha^{\beta}\right)$, obeyed (7) and the following relation,

$$
\sum_{i=1}^{n} d_{i}\left(\alpha^{i}\right)^{2}+\alpha^{\beta} \alpha^{\gamma} h_{\beta \gamma}=1
$$

instead of (8). The conditions under which this asymptotic Kasner behavior of the metric and scalar field occurred were formulated in terms of brane $U^{s}$ covectors,

$$
U^{s}(\alpha) \equiv U_{A}^{s} \alpha^{A} \equiv \sum_{i \in I_{s}} d_{i} \alpha^{i}-\chi_{s} \lambda_{a_{s} \gamma} \alpha^{\gamma},
$$

with the scalar products being positive definite,

$$
\left(U^{s}, U^{s}\right)=d_{s}\left(1+\frac{d_{s}}{2-D}\right)+\lambda_{a_{s} \alpha} \lambda_{a_{s} \beta} h^{\alpha \beta}>0 .
$$

Namely, one obtained asymptotic Kasner-type behavior if for all branes [with $\left(U^{s}, U^{s}\right)>0$ ]

$$
U^{s}(\alpha)>0 .
$$

In the above, $d_{s}$ is the dimension of the brane worldvolume manifold, $a_{s} \in \Delta$ is a color index for the brane, and $\chi_{s}=+1,-1$ for an electric or a magnetic brane, respectively. Here for any brane labeled by index $s$ the set $I_{s}=\left\{i_{1}, \ldots, i_{k}\right\}, i_{1}<\ldots<i_{k}$, describes the location of the brane, i.e. the brane is "attached" to the submanifold $M_{s}=M_{i_{1}} \times \ldots \times M_{i_{k}}$. The vectors $u_{i}^{(\nu)}$ and $U_{A}^{s}$ play similar roles for the respective cases of perfect-fluid sources and brane sources. In the case when the condition (16) was not satisfied, namely, if for any Kasner set of parameters, $\alpha=\left(\alpha^{A}\right)$, there existed a brane with an index $s_{0}$ [obeying $\left.\left(U^{s_{0}}, U^{s_{0}}\right)>0\right]$, such that

$$
U^{s_{0}}(\alpha) \leq 0,
$$

one obtained never-ending oscillating behavior near the singularity. 
It should be stressed that the billiard approach of [5] was derived in a class of block-diagonal metrics defined on the product of Einstein spaces (1) with certain restrictions imposed on the signatures of the Einstein metrics, $g^{(i)}$. These restrictions are similar to the conditions $\rho^{(\nu)}>0$ for $\left(u^{(\nu)}\right)^{2}>0$ in the multicomponent fluid case.

In [18] Damour and Henneaux conjectured that the never-ending oscillating behavior is generic to a certain class of string theory inspired cosmologies (see also [19] for a review). Moreover, the authors of [18] give a sketch of a proof of this conjecture. Although the ansatz for the cosmological metric in [18] contained $d$-beins leading to additional gravitational walls in the billiards and the Kasner parameters also depended on spatial coordinates, the analysis of [18], in essence, deals with the Bianchi-I type model with a maximal number of composite electric branes that form the billiard walls according to the prescription of [5]. The gravitational walls are irrelevant for the proof in [18]. The key point of this proof is the verification of inequalities (17) for the maximal set of electric branes. [In [18] the coefficients $U_{A}^{s}$ in the linear functions $U^{s}(\alpha)=U_{A}^{s} \alpha^{A}$ were called electric and magnetic exponents.] This verification was carried out for $D=$ 11 supergravity ( $M$ theory), and also for the four $D=10$ string theories - types IIA, IIB, I, and heterotic $(H)-$ using dualities.

In their paper [18] the authors did not sketch a proof of their conjecture for pure cosmological nondiagonal metric with the line element of the form

$$
d s^{2}=-\exp (2 \gamma(t)) d t^{2}+g_{i j}(t) d y^{i} d y^{j} .
$$

In essence the authors in [18] dealt with the pure cosmological diagonal Bianchi-I type metric [the nonhomogeneity of the metric and the presence of $(D-1)$-beins were irrelevant for the proof] but they did not take into consideration the $(D-1)(D-2) / 2$ equations coming from the nondiagonal part of Hilbert-Einstein equations.

This naturally leads to the question as to whether the conjecture of Damour and Henneaux may be reformulated somehow for composite $S$-brane solutions with diagonal metrics. At present we have no examples of solutions in string inspired cosmologies with never-ending oscillating behavior near the singularity when the metric is diagonal.

In this paper we point out that a certain class of composite $S$-brane solutions with diagonal metrics can avoid this oscillating behavior near the singularity even if the diagonal part of Hilbert-Einstein equations leads to "confining" billiards.

In Sec. II we give the general set up of our system of $(D=n+1)$-dimensional gravity with the $(p+2)$-form field. We consider a composite $S p$-brane ansatz for the antisymmetric $(p+2)$-form field, with all the ansatz functions depending only on the time coordinate. An important feature of the composite ansatz is that the charge densities of the branes obey certain quadratic constraints $[16,17]$. In
[16] it was found that these constraints have "maximal" solutions with all nonzero electric brane charge densities in specific odd dimensions with specific rank of the form fields (i.e. $D=5,9,13 \ldots$ and $p=1,3,5 \ldots$, respectively). In Sec. II we review these solutions for the model of [16] but without the scalar field.

In Sec. III we give two explicit examples of how the oscillating behavior near the singularity is stopped when one replaces noncomposite $S$ branes (noncomposite usually means that for one form there is no more than one brane) with the composite $S$ branes. The two examples both have diagonal metrics, and spacetime dimension and $(p+2)$ forms given by $D=4, p=0$ and $D=5, p=1$, respectively.

\section{II. $D$-DIMENSIONAL GRAVITY WITH $(p+2)$ FORM}

The multidimensional system considered here is similar to that of [16] but without the scalar field. The action is given by

$$
S=\int_{M} d^{D} z \sqrt{|g|}\left[R[g]-\frac{1}{q !} F_{[q]}^{2}\right],
$$

where $g$ is the metric with the line element $g_{M N} d z^{M} d z^{N}$ $(M, N=1, \ldots, D)$, and

$$
F_{[q]}=d A_{[q-1]}=\frac{1}{q !} F_{M_{1} \ldots M_{q}} d z^{M_{1}} \wedge \ldots \wedge d z^{M_{q}}
$$

is a $q$ form $(q=p+2 \geq 2)$ on a $D$-dimensional manifold $M$. The manifold is of the form $M=\left(t_{-}, t_{+}\right) \times \mathbf{R}^{n}$ with the first coordinate $t$ being the distinguished, timelike coordinate. The metric is diagonal and of the form

$$
d s^{2}=-\mathrm{e}^{2 \gamma(t)} d t^{2}+\sum_{i=1}^{n} \mathrm{e}^{2 \phi^{i}(t)}\left(d y^{i}\right)^{2} .
$$

The ansatz functions $\gamma(t), \phi^{i}(t)$, and the $q$ forms are assumed to depend only on the distinguished, timelike coordinate $t$. The functions $\gamma, \phi^{i}$ are smooth.

The $(q=p+2)$ form, $F$, is taken in the electric composite $S p$-brane ansatz of the form

$$
F=\sum_{I \in \Omega_{e}} d \Phi^{I} \wedge \tau(I)
$$

where $\Phi^{I}$ is a scalar potential. This ansatz is composite since it is a sum of monomial terms rather than a single term for the noncomposite case. In (22) $\Omega_{e}$ is the set of multi-indices having the form $\left\{i_{0}, i_{1}, \ldots, i_{p}\right\}$ (all $i_{k}$ are among the numbers $1, \ldots, n$ ).

In (22) we have introduced a volume form of rank $d(I)=p+1$,

$$
\tau(I) \equiv d y^{i_{0}} \wedge \ldots \wedge d y^{i_{p}} .
$$

Here $I=\left\{i_{0}, \ldots, i_{p}\right\}$ which corresponds to the brane submanifold with the coordinates $y^{i_{0}}, \ldots, y^{i_{p}}$. 
The scalar potential depends only on the distinguished, timelike coordinate, i.e. $\Phi^{I}=\Phi^{I}(t)$. The solution of the "Maxwell" equations for the scalar potentials $\Phi^{I}(t)$ has the following form [16],

$$
\dot{\Phi}^{I}=Q_{I} \exp \left(2 U^{I}\right), \quad U^{I}=U^{I}(\phi)=\sum_{i \in I} \phi^{i},
$$

where $Q_{I}$ are constant charge densities and $I \in \Omega_{e}$. The $q=(p+2)$ form then reads

$$
\begin{aligned}
F_{[q]} & =\frac{1}{(p+1) !} Q_{i_{0} \ldots i_{p}} \exp \left(2 \phi^{i_{0}}+\ldots+2 \phi^{i_{p}}\right) d u \\
& \wedge d y^{i_{0}} \wedge \ldots \wedge d y^{i_{p}}
\end{aligned}
$$

where $Q_{i_{0} \ldots i_{p}}$ are components of antisymmetric form coinciding with $Q(I)$ for $i_{0}<\ldots<i_{p}$.

Since the Ricci tensor is diagonal one finds

$$
F_{i M_{2} \ldots M_{q}} F_{j}^{M_{2} \ldots M_{q}} \propto T_{i j}=0, \quad i \neq j,
$$

where $T_{i j}$ is the stress-energy tensor. This leads to constraints among the charge densities, $Q_{i_{0} \ldots i_{p}}$. Absorbing the functional dependence on $t$ into the definition of the charge densities as $\bar{Q}_{i_{0} \ldots i_{p}}$ via

$$
\bar{Q}_{i_{0} \ldots i_{p}} \equiv Q_{i_{0} \ldots i_{p}} \prod_{k=0}^{p} \exp \left(\phi^{i_{k}}\right),
$$

one can write the constraints as [16]

$$
C_{i}^{j} \equiv \sum_{i_{1}, \ldots, i_{p}=1}^{n} \bar{Q}_{i i_{1} \ldots i_{p}} \bar{Q}^{j i_{1} \ldots i_{p}}=0,
$$

$i \neq j, i, j=1, \ldots, n$. Here the indices are lifted by the flat Euclidean metric $\left(\eta_{a b}\right)=\left(\eta^{a b}\right)=\operatorname{diag}(1, \ldots, 1)$. These $\bar{Q}_{i_{0} \ldots i_{p}}$ can be viewed as "running" charge densities with the functional dependence coming from $\phi^{i_{k}}(t)$.

In [16] it was shown that for

$$
D=4 m+1, \quad p=2 m-1,
$$

these constraints could be satisfied if the nonrunning charge densities were self-dual or anti-self-dual in a flat Euclidean space $\mathbf{R}^{n}$, i.e.

$$
Q_{i_{0} \ldots i_{p}}= \pm \frac{1}{(p+1) !} \varepsilon_{i_{0} \ldots i_{p} j_{0} \ldots j_{p}} Q^{j_{0} \ldots j_{p}}= \pm(* Q)_{i_{0} \ldots i_{p}},
$$

and if all the scale factors were the same

$$
\phi^{i}(t)=\phi(t) .
$$

In (30) $\varepsilon_{i_{0} \ldots i_{p} j_{0} \ldots j_{p}}$ is the completely antisymmetric symbol.

Along the lines of [16] (when a scalar field was present) the equations of motion for the action (19) have the following solution when relations (29) are satisfied:

$$
\begin{gathered}
\phi(t)=\frac{2}{n} f(t), \quad f(t)=-\ln \left[\left(t-t_{0}\right)\left|Q^{2} K\right|^{1 / 2}\right], \\
d s^{2}=-e^{2 n \phi(t)} d t^{2}+e^{2 \phi(t)} \sum_{i=1}^{n}\left(d y^{i}\right)^{2} \\
F=e^{2 f(t)} d t \wedge Q \\
Q=\frac{1}{(p+1) !} Q_{i_{0} \ldots i_{p}} d y^{i_{0}} \wedge \ldots \wedge d y^{i_{p}},
\end{gathered}
$$

where $K=-\frac{n}{4(n-1)}$ and $Q^{2}=\sum_{I \in \Omega_{e}} Q^{2}(I)$. In the above the ansatz function $\gamma(t)=\sum_{i=1}^{n} \phi^{i}(t)=n \phi(t)$ because of (31). The charge density form $Q$ is of rank $n / 2=2 m$ and is self-dual or anti-self-dual in a flat Euclidean space $\mathbf{R}^{n}$ : $Q= \pm * Q$. This solution may be obtained from that of Ref. [16] by taking the parameters as $C=C_{1}=C_{2}=$ $\lambda=0$. Note that this solution with composite form field does not have oscillating Kasner-like behavior as one approaches the singularity at $t \rightarrow+\infty$ (or $\tau \rightarrow+0$ ).

It should be stressed that in the case $D=5$ and $p=1$, the solution presented above is a general one when all charge densities $Q(I)$ are nonzero, $I \in \Omega_{e}$. More, rigorously, here as in [16], we get that the running "charge densities" $\bar{Q}_{i_{0} i_{1}}$ are self-dual or anti-self-dual and all scale factors are the same up to constants

$$
\phi^{i}(t)=\phi(t)+c^{i},
$$

$i=1, \ldots, 4$. The constants $c^{i}$ may be absorbed by an appropriate rescaling of $y^{i}$ coordinates.

\section{BREAKING OF OSCILLATING BEHAVIOR NEAR THE SINGULARITY BY CONSTRAINTS}

In [5] it was found that certain cosmological models with $p$ branes may have a "never-ending" oscillating behavior, i.e. as one approaches the cosmological singularity the Kasner parameters of the asymptotic metric "jump" between different locally constant values. This is similar to the behavior found in the Bianchi-IX model [8] and in multidimensional models with multicomponent perfect fluid [1-3].

This oscillating behavior may be described graphically using the so-called billiard representation near the singularity. The point described by the anisotropic part of the minisuperspace coordinates $z=\left(z^{a}\right)(a=1, \ldots, n-1)$ moves in the asymptotical regime near the singularity in a region enclosed by "hard walls" corresponding to inequalities on the Kasner parameters such as (16). The $z^{a}$ are linear combinations of the $x^{i}(t)$ from (2), i.e. they are combinations of logarithms of the scale factors. The point $z(t)$ traces out a path in this region until it encounters a wall at which point it recoils and moves toward another wall. An example of the billiard approach for a model with scalar fields and form fields (with the form fields having a composite $S$-brane ansatz) can be found in [5]. 
Here we show that this oscillating behavior near the singularity can be broken in certain cases due to the compositeness of the branes and the diagonality of the metric. We show this via two examples: $D=4$ with 2 forms, and $D=5$ with 3 -forms. In each case the breaking of the oscillating behavior is a result of the constraints on charge densities, following from the nondiagonal part of Hilbert-Einstein equations.

\section{A. 4-dimensional model with 2-form}

Let us consider the 4-dimensional model

$$
S_{4}=\int_{M} d^{4} z \sqrt{|g|}\left[R[g]-\frac{1}{2 !} F_{[2]}^{2}\right],
$$

with the metric $g$ and 2-form $F_{[2]}=d A_{[1]}$ defined on a 4manifold $M$. The metric is taken as diagonal and of the form

$$
d s^{2}=-\mathrm{e}^{2 \gamma(t)} d t^{2}+\sum_{i=1}^{3} \mathrm{e}^{2 \phi^{i}(t)}\left(d y^{i}\right)^{2}, \quad \text { with } \quad \gamma=\sum_{i=1}^{3} \phi^{i} .
$$

The electric 2-form has the composite form

$$
F_{[2]}=\sum_{i=1}^{3} d \Phi^{i} \wedge d y^{i}
$$

From (24) we get

$$
\dot{\Phi}^{i}=Q_{i} \exp \left(2 \phi^{i}\right)
$$

$i=1,2,3$.

For this case the constraints from (28) are

$$
C_{i j}=Q_{i} Q_{j}=0,
$$

$i \neq j ; i, j=1,2$, 3. It follows from (40) that only one charge $Q_{i_{0}}$, may be nonzero. At the end of this section we will use the billiard representation to show that this implies one has asymptotic Kasner behavior, rather than never-ending oscillating behavior near the singularity.

Now let us omit for a moment the constraints (40), i.e. the nondiagonal part of Einstein equations is not considered. Such a "truncation" is equivalent to considering a model with three 2 -forms, having the following action,

$$
S_{4 b r}=\int_{M} d^{4} z \sqrt{|g|}\left[R[g]-\frac{1}{2 !} \sum_{i=1}^{3}\left(F_{[2]}^{i}\right)^{2}\right],
$$

instead of (36) and with a noncomposite ansatz

$$
F_{[2]}^{i}=d \Phi^{i} \wedge d y^{i},
$$

$i=1,2,3$, instead of (38). Relation (39) survives in this noncomposite case.

The asymptotical behavior near the singularity $(\tau \rightarrow$ $+0)$ for this truncated or noncomposite model with nonzero charges is described by the metric [5]

$$
d s_{a s}^{2}=-d \tau^{2}+\sum_{i=1}^{n} A_{i} \tau^{2 \alpha^{i}(\tau)}\left(d y^{i}\right)^{2},
$$

with the Kasner parameters satisfying the relations

$$
\sum_{i=1}^{n} \alpha^{i}=\sum_{i=1}^{n}\left(\alpha^{i}\right)^{2}=1 .
$$

$A_{i}>0$ are constants and $n=3$. The Kasner "parameters" $\alpha^{i}(\tau)$ are constant in the intervals $\tau \in\left[\tau_{k+1}, \tau_{k}\right), k=$ $0,1,2, \ldots$, and $\tau_{k} \rightarrow+0$ as $k \rightarrow \infty$, but take different values for neighboring intervals.

Thus, one gets never-ending oscillating behavior near the singularity, equivalent to that of the Bianchi-IX model [8]. The dynamics of this "motion" may be described graphically as the motion of a "particle" in the well-known triangle billiard "table" belonging to Lobachevsky space [9] (see also [3,10-12]). The reflection from a wall describes the change to a different Kasner "epoch." The "collision law" for the particle in terms of Kasner parameters may be obtained from general relations given in $[18,20]$. This triangular billiard table is depicted in Fig. 1. It is the interior triangle with curved, hard walls labeled by $+\infty$. These infinite positive walls result from the fact that the scalar product of all $U$ vectors obeys

$$
\left(U^{I}, U^{I}\right) \equiv G^{i j} U_{i}^{I} U_{j}^{I}=\frac{1}{2}>0,
$$

for $I=\{i\}, i=1,2,3$ [3,5]. Relation (45) is obtained from (15) with $d_{s}=1$ and $h^{\alpha \beta}=0$. Here and in the next sub-

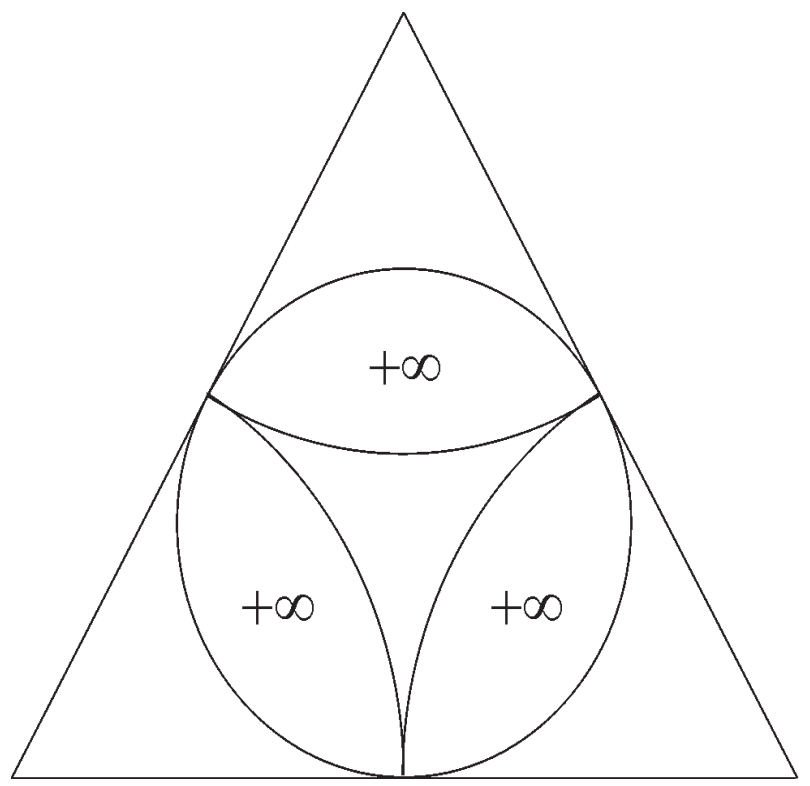

FIG. 1. The triangle billiard for $D=4, q=2$. The hard walls, $+\infty$, come from (45). The particle described by the minisuperspace coordinates, $z^{a}$, moves in this billiard until it encounters a wall and is reflected. The enclosure of the billiard is noncompact but the area is finite. 
section $G^{i j}=\delta_{i j}+\frac{1}{2-D}$ are the components of the inverse matrix to the matrix of the minisuperspace metric $G_{i j}=$ $\delta_{i j}-1$ with $i, j=1, \ldots, n$ (for its diagonalization, see, for example, [21]). The enclosure of the billiard table in Fig. 1 is not compact but it has a finite area.

The never-ending oscillating behavior for the noncomposite model takes place since for any Kasner set $\alpha=\left(\alpha^{i}\right)$ there exists a brane $U$ vector satisfying

$$
U^{I}(\alpha)=\alpha^{i} \leq 0,
$$

for $I=\{i\}, i=1,2,3$ [5]. We note that the equality in the right-hand side of (46) takes place at the three vertices of the triangle, namely, $\alpha=(0,0,1),(0,1,0),(1,0,0)$.

Returning to the composite model, the constraints (40) imply that two of the charges vanish and, hence, two of the walls of the billiard table vanish. This opens a region at infinity for a nonoscillating asymptotical Kasner-type behavior.

This example has a straightforward generalization to $(n+1)$ dimensions. It is interesting to note that as it was shown recently in [22] in the general case of nondiagonal metric (18) the oscillating behavior near the singularity is recovered for the $(n+1)$-dimensional model with a 2form. In this case there is (asymptotically) one "jumping" wall that effectively acts as a full set of walls.

\section{B. $D=5$ model with 3-form}

Now we consider the following 5-dimensional model,

$$
S_{5}=\int_{M} d^{5} z \sqrt{|g|}\left[R[g]-\frac{1}{3 !}\left(F_{[3]}\right)^{2}\right]
$$

with metric $g$ and 3-form $F_{[3]}=d A_{[2]}$ defined on a 5dimensional manifold $M$.

It follows from the results of Sec. II that the general solutions with maximal number of composite electric $S 1$ branes (strings) resulting from the action (47) with a diagonal metric are

$$
d s^{2}=-\mathrm{e}^{8 \gamma(t)} d t^{2}+\sum_{i=1}^{4} \mathrm{e}^{2 \phi(t)+2 c^{i}}\left(d y^{i}\right)^{2},
$$

and

$$
F_{[3]}=\sum_{I} d \Phi^{I} \wedge \tau(I),
$$

where $I=\{i, j\}, i<j ; i, j=1,2,3,4$, and all charges $Q_{I}$ are nonzero. As can be seen from (32)-(34) this solution has neither an oscillating nor even a Kasner-type behavior [i.e. writing the metric in Kasner form, (43), the Kasner powers do not satisfy (44)] near the singularity.

Now we omit the constraints (28). This leads to a model with six 3-forms having the action

$$
S_{5 b r}=\int_{M} d^{5} z \sqrt{|g|}\left[R[g]-\frac{1}{3 !} \sum_{I}\left(F_{[3]}^{I}\right)^{2}\right]
$$

instead of (47) and a noncomposite ansatz

$$
F_{[3]}^{I}=d \Phi^{I} \wedge \tau(I),
$$

instead of the composite one (49).

We can use the results of [5] to show that for these noncomposite solutions with nonzero charge densities, $Q_{I}$, of the 1-branes (strings) we get never-ending oscillating behavior near the singularity as $\tau \rightarrow+0$, described by the metric formulas (43) and (44) with $n=4$ and certain locally constant functions $\alpha^{i}(\tau)$.

In this case we get a 3-dimensional billiard table with six walls that form a deformed cube. From the relationships in [5] the explicit form of this billiard belonging to the 3dimensional Lobachevsky space ("ball") $H^{3}=D^{3}=$ $\left\{\vec{z} \in \mathbf{R}^{3}:|\vec{z}|<1\right\}$ is given by the six inequalities

$$
\left|\vec{z}-\vec{v}_{k}\right|>\sqrt{2}
$$

$k=1, \ldots, 6$, where

$$
\begin{gathered}
\vec{v}_{1}=-\vec{v}_{6}=(\sqrt{3}, 0,0), \quad \vec{v}_{2}=-\vec{v}_{5}=(0, \sqrt{3}, 0), \\
\vec{v}_{3}=-\vec{v}_{4}=(0,0, \sqrt{3}) .
\end{gathered}
$$

As in the previous example the enclosure of the billiard is not compact, but the volume is finite. This can be seen in terms of the "illumination" of the Kasner sphere by point sources of "light." The sources of light located at the points $\vec{v}_{k}, k=1, \ldots, 6$, completely illuminate the unit sphere $S^{2}=\left\{\vec{z} \in \mathbf{R}^{3} \| \vec{z} \mid=1\right\}$, and according to the illumination theorem of $[3,5]$ the billiard has a finite volume.

As in the previous example the walls come from the fact that the scalar products of all $U$ vectors obey

$$
\left(U^{I}, U^{I}\right) \equiv G^{i j} U_{i}^{I} U_{j}^{I}=\frac{2}{3}>0,
$$

for $I=\{i, j\}, i<j[3,5]$. In (54) we have used (15) with $d_{s}=2$ and $h^{\alpha \beta}=0$.

One will get never-ending oscillating behavior for the noncomposite model since for any Kasner set, $\alpha=\left(\alpha^{i}\right)$, there exists a brane $U$ vector which satisfies

$$
U^{I}(\alpha)=\alpha^{i}+\alpha^{j} \leq 0,
$$

for $I=\{i, j\}, i<j$. We note that the equality in the righthand side of (55) takes place for $\alpha=$ $(0,0,0,1),\left(\frac{1}{2}, \frac{1}{2}, \frac{1}{2},-\frac{1}{2}\right)$ and 6 other sets obtained by permutations. These are points on the Kasner sphere $S^{2}$ coinciding with the eight vertices of the "deformed" cube.

In the composite case, as we have seen from the exact solution given by (32)-(34), there is no oscillating behavior of the scale factors. From the billiard representation point of view this solution corresponds to a static point in the billiard. 


\section{CONCLUSIONS}

In this paper we have presented examples showing that the never-ending oscillating behavior near the singularity with diagonal metric can be broken when noncomposite branes are replaced by composite ones.

The mechanisms of breaking the oscillating behavior are different in these examples. In the 4D case the "constraints" (28) destroy all the walls of the billiard table except one. In the 5D case, in general, some of the walls could also be "destroyed" due to zero charge densities. But in our example all walls survive since all the charge densities $Q_{i j}$ are nonzero and obey (anti)-self-duality relations. In this case the constraints select only those solutions that have simple isotropic behavior (with non-Kasner and nonoscillating behavior near the singularity).

These examples suggest a conjecture that an analogous effect may take place for 11-dimensional supergravity and 10-dimensional models of superstring origin. In other words, one may consider the following hypothesis: for certain supergravity models (say, for $D=11$, or $D=10$ IIA supergravities) there are no composite $S$-brane solutions with (block-) diagonal metrics that have a neverending oscillating behavior near the singularity.

We note that in [5] an example of oscillating behavior described by a 4-dimensional billiard of finite volume in a "truncated" $D=11$ supergravity model (without the Chern-Simons term) was suggested. This billiard was supported by ten magnetic 5-branes. It was shown that the inclusion of the Chern-Simons term destroyed some walls of the billiard table and allowed an asymptotical Kasnerlike behavior as $\tau \rightarrow+0$. Interestingly, the constraints of Chern-Simons origin in [5] look very similar to the constraints considered in this paper. It should be pointed out that recently some other mechanisms for avoiding oscillating behavior near the singularity were suggested in $[23,24]$.

The main conclusion of this paper is that the neverending oscillating behavior near the singularity of solutions in certain cosmological models with forms (and scalar fields) can be broken or avoided. This avoidance of the oscillating behavior can be attributed to the form of the ansatz considered here: a diagonal metric, and composite electric ansatz for the form fields. This result comes from the constraints on the brane charge densities which are due to the diagonality of the metric and the compositeness of the brane system. This shows that such behavior may not be generic to such higher dimensional cosmologies.

\section{ACKNOWLEDGMENTS}

The work of V.D. I. and V. N. M. was supported in part by DFG Grant No. 436 RUS 113/807/0-1 and by the Russian Foundation for Basic Researches, Grant No. 0502-17478. D. S. would like to thank Professor Max Chaves for the invitation to work at the Universidad de Costa Rica.
[1] V. D. Ivashchuk, A. A. Kirillov, and V. N. Melnikov, Izv. Vuzov (Fizika) 11, 107 (1994) [Russian Physics Journal 37, 1102 (1994)].

[2] V.D. Ivashchuk, A. A. Kirillov, and V. N. Melnikov, Pis'ma Zh. Eksp. Teor. Fiz. 60, 225 (1994) [JETP Lett. 60, 235 (1994).

[3] V.D. Ivashchuk and V.N. Melnikov, Classical Quantum Gravity 12, 809 (1995).

[4] V. D. Ivashchuk and V. N. Melnikov, R\&C Dynamics 1, 23 (1996).

[5] V. D. Ivashchuk and V. N. Melnikov, J. Math. Phys. (N.Y.) 41, 6341 (2000).

[6] H. Dehnen, V.D. Ivashchuk, and V. N. Melnikov, Gen. Relativ. Gravit. 36, 1563 (2004).

[7] C. W. Misner, Phys. Rev. 186, 1319 (1969).

[8] V. A. Belinskii, E. M. Lifshitz, and I. M. Khalatnikov, Usp. Fiz. Nauk 102, 463 (1970) (in Russian); Adv. Phys. 31, 639 (1982).

[9] D. M. Chitre, Ph.D. thesis, University of Maryland, 1972.

[10] J. Pullin, "Time and Chaos in General Relativity," Syracuse University Report No. Su-GP-91/1-4 (to be published); in Proceedings of SILARG VII Cocoyos, Mexico, 1990, edited by J.C. D'Olivo (World Scientific, Singapore, 1991).
[11] A. A. Kirillov, Zh. Eksp. Teor. Fiz. 55, 561 (1992) (in Russian).

[12] A. A. Kirillov, Int. J. Mod. Phys. D 3, 431 (1994).

[13] C. W. Misner, "The Mixmaster Cosmological Metrics," Report No. UMCP PP94-162, 1994 (to be published).

[14] A. A. Kirillov and V. N. Melnikov, Phys. Rev. D 52, 723 (1995).

[15] K. Behrndt and S. Forste, Nucl. Phys. B430, 441 (1994); hep-th/9312167; K. Behrndt, S. Forste, and S. Schwager, Nucl. Phys. B508, 391 (1997).

[16] A. Lukas, B. A. Ovrut, and D. Waldram, Phys. Lett. B 393, 65 (1997).

[17] H. Lü, S. Mukherji, C. N. Pope, and K.-W. Xu, Phys. Rev. D 55, 7926 (1997).

[18] H. Lü, S. Mukherji, and C. N. Pope, Int. J. Mod. Phys. A 14, 4121 (1999).

[19] K. A. Bronnikov, M. A. Grebeniuk, V. D. Ivashchuk, and V. N. Melnikov, Gravitation Cosmol. 3, 105 (1997).

[20] M. A. Grebeniuk, V.D. Ivashchuk, and V. N. Melnikov, Gravitation Cosmol. 3, 243 (1997).

[21] V. D. Ivashchuk and V. N. Melnikov, J. Math. Phys. (N.Y.) 39, 2866 (1998).

[22] V. D. Ivashchuk and S.-W. Kim, J. Math. Phys. (N.Y.) 41, 444 (2000). 
[23] V.D. Ivashchuk and V.N. Melnikov, Classical Quantum Gravity 18, R87 (2001).

[24] M. Gutperle and A. Strominger, J. High Energy Phys. 04 (2002) 018.

[25] C. M. Chen, D. M. Gal'tsov, and M. Gutperle, Phys. Rev. D 66, 024043 (2002).

[26] M. Kruczenski, R. C. Myers, and A. W. Peet, J. High Energy Phys. 05 (2002) 039.

[27] S. Roy, J. High Energy Phys. 08 (2002) 025.

[28] N. S. Deger and A. Kaya, J. High Energy Phys. 07 (2002) 038.

[29] V.D. Ivashchuk, Classical Quantum Gravity 20, 261 (2003).

[30] N. Ohta, Phys. Lett. B 558, 213 (2003).

[31] V. D. Ivashchuk, hep-th/0309027.

[32] V.D. Ivashchuk, in Proceedings of the 18th Course of the School on Cosmology and Gravitation: The Gravitational Constant. Generalized Gravitational Theories and Experiments, Erice, 2003, edited by G. T. Gillies, V. N.
Melnikov, and V. de Sabbata (Kluwer Academic Publishers, Dordrecht, 2004), pp. 39-64.

[33] V. D. Ivashchuk and D. Singleton, J. High Energy Phys. 10 (2004) 061.

[34] V.D. Ivashchuk and V. N. Melnikov, Classical Quantum Gravity 14, 3001 (1997); 15, 3941(E) (1998).

[35] T. Damour and M. Henneaux, Phys. Rev. Lett. 85, 920 (2000).

[36] T. Damour, M. Henneaux, and H. Nicolai, Classical Quantum Gravity 20, R145 (2003).

[37] V. D. Ivashchuk, V. N. Melnikov, and A. I. Zhuk, Nuovo Cimento B 104, 575 (1989).

[38] R. Benini, A. A. Kirillov, and G. Montani, Classical Quantum Gravity 22, 1483 (2005).

[39] J. K. Erickson, D. H. Wesley, P. J. Steinhardt, and N. Turok, Phys. Rev. D 69, 063514 (2004).

[40] D. Wesley, P. J. Steinhardt, and N. Turok, Phys. Rev. D 72, 063513 (2005). 